\title{
RESEARCH ON URBAN CONSTRUCTION LAND CHANGE DETECTION METHOD BASED ON DENSE DSM AND TDOM OF AERIAL IMAGES
}

\author{
X. Zhu ${ }^{1, *}$, G. Pang ${ }^{2, *}$, P. Chen ${ }^{1}$, Y. Tao ${ }^{1}$, Y. Zhang ${ }^{1}$, X. Zuo ${ }^{1}$ \\ ${ }^{1}$ Beijing Institute of Surveying and Mapping, 100038 Beijing, P. R. China - (zhuxk, cpx, taoyc, zhangyr, zuoxg)@bism.cn \\ ${ }^{2}$ Beijing Tracesky Ltd. Company, 101104 Beijing, P. R. China - gfpang@sina.com
}

KEY WORDS: Urban Construction Land, Change Detection, Digital Surface Model (DSM), TDOM (True Digital Orthophoto Map), Difference of DSM, Aerial Images

\begin{abstract}
:
The scale of urban construction land is an important factor of the process of urbanization. This paper presented an urban construction land change detection method combining dense DSM difference and TDOM tree extraction of traditional aerial photos: the pixel-level DSM and TDOM for two period are obtained by Semi-Global Matching (SGM) and Multi View Stereo (MVS), respectively. After DSM pre-processing including noise filter and hole filling, the difference of DSM is calculated. The segmentation and tree extraction of TDOM are proposed to reduce the change errors caused by the crown influence of different seasons. Based on this method, 2 experiments were carried out. One was for the urban construction land located at 5th ring road south in Beijing with ADS80 aerial images obtained in 2016 and 2017, and the other was for the building demolition and construction of Dongcheng and Xicheng District of Beijing with UltraCam, the frame camera and RCD30 between 2015 and 2017. Through the experiments, it was concluded that the result of the method could identify all the changes both in the plane and in the elevation, and the edges of the change patches were clear and regular, which could assist checking the manual change extraction results. The minimum area of the change patches could be $5 \mathrm{~m} 2$ with the DSM resolution of $0.2 \mathrm{~m}$, which was of great significance for the urban construction change detection including the factory, residential area and also the urban regional greening.
\end{abstract}

\section{INTRODUCTION}

Nowadays, Modern cites gather population, economics and natural resources with locational advantage and urbanization becomes an important socio-economic phenomenon. There are always frequent construction activities in urban area and the scale of urban construction land is an important factor of the process of urbanization. Built-up areas, as one of the main types of land cover in urban, directly reflects the activities of human beings and level of urban construction. Its changes have a nonnegligible impact on urban economic development, resource utilization, and ecological and environmental protection as well, especially for Beijing, the capital of China. Therefore, the dynamic monitoring of urban built-up areas at regional scale is of great value for understanding the urban development process, assessing the level of urban land use, and formulating more reasonable urban planning strategies.

Remote sensing has become one of the main technical approaching in monitoring land-use dynamics with the resolution from $0.5 \mathrm{~m}$ to $250 \mathrm{~m}$, the application of remote sensing data can effectively extract urban built-up areas on a regional or larger scale, and studying the changes is of great significance for monitoring the evolution of urban spatiotemporal pattern. As the spaceborne remote sensing techniques develop, multisource satellite data area applied to monitor the urban construction land: Huang et al. (2016) combined the MODIS and DMSP/OLS nighttime data to extract urban built-up area. Wang (2018) monitored the dynamics of built-up area of Jiangxi Province for 20 years with Landsat and DMSP/OLS nighttime data.
It is worthy noting that the change of urban construction land can be detected not only in plane, but also in elevation. Except large scale spaceborne monitoring, 3D change detection with airborne data is available for urban management with photogrammetry. Digital Surface Model (DSM) recorded urban surface including both terrain and residential and vegetation information (Liu et al., 2002; Meng et al., 2018). The difference between two well matched DSM represents the urban surface elevation changes, which are always in accordance with plane changes.

In urban areas, with the development of urban construction, a large number of illegal buildings have appeared, and the existing monitoring mainly depends on the ground inspection, spaceborne remote sensing and so on. The existing methods do not meet the requirements because of long monitoring period, large amount of field work, and limited small change patches detection ability. Wu et al. (2018) proposed Unmanned Aerial Vehicle (UAV) oblique photography to monitor the urban illegal building. Oblique photography technology could produce high precision DSM, Digital Orthophoto Map (DOM), 3D model and other spatial data, which realizes quantitive monitoring of illegal buildings with 3D information. With the DSM difference, automatic extraction and quantitative analysis of illegal buildings were realized. Peng et al. (2015) presented an algorithm of combining LiDAR data and original aerial imagery for 3D building change detection.

Normally, there are some pseudo changes in the detection result with DSM caused by seasonal difference, combining both elevation and plane information is helpful to filer the

\footnotetext{
* Corresponding author
} 
pseudo changes. DOM is usually applied to identify the changes caused by seasonal vegetation difference (Feng et al., 2016). However, the elevation displacement and shadows of DOM may introduce new errors. The True Digital Orthophoto Map (TDOM) with oblique photography is better (Liu et al., 2018). With the development of pixel-level matching technology and high performance computing, especially the application of Semi-Global Matching (SGM) (Shao, 2019) and Multi View Stereo (MVS) algorithms, traditional digital aerial photographs (non tilt or non large overlap) could also be applied to extract the DSM and TDOM with the same accuracy as those of LiDAR and tilted photograph. In this paper an change detection workflow for urban area combining DSM and TDOM of traditional aerial photos is proposed.

\section{MEHODOLOGY}

With the dense DSM and TDOM extracted, this paper presented an urban construction land change detection method based on DSM difference and TDOM vegetation recognition, the work flow is listed in Figure 1:

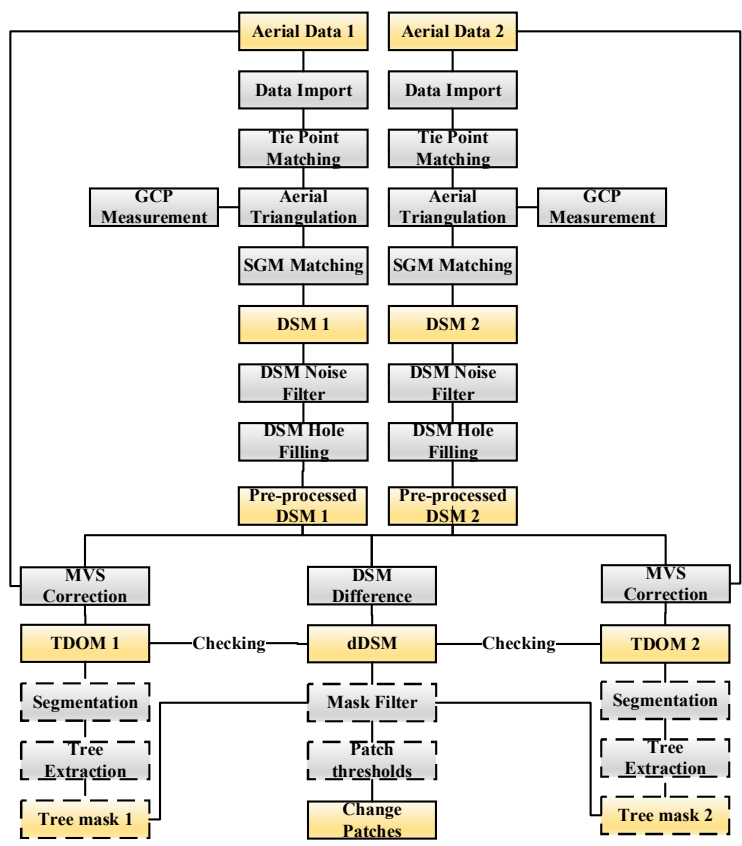

Figure 1. Urban construction land change detection work flow

There are three main steps in the work flow:

1) DSM and TDOM acquisition. The pixel-level DSM data of two traditional digital aerial photographs can be obtained by tie point matching, aerial triangulation, Ground Control Points (GCPs) measurement, SGM matching. In Figure 1, DSM 1, DSM 2 represent two temporal DSM results, respectively. Considering there are a few flying points and holes in the DSM obtained by automatic matching, the DSM needs to be preprocessed before the change detection step. The dense matching points greater than 5-10 times the average elevation and negative values are checked by visual identification, and the noises are filtered through threshold setting. The holes on DSM are usually caused by insufficient overlap, shadows, occlusion and other factors. The hole filling step of DSM mainly uses the lowest value of surrounding filling to ensure the perpendicular characteristics of sides of buildings. Considering there is no elevation displacement in TDOM compared with DOM (Figure 2), TDOM data corrected by MVS correction with DSM assist to check the difference result of DSM.
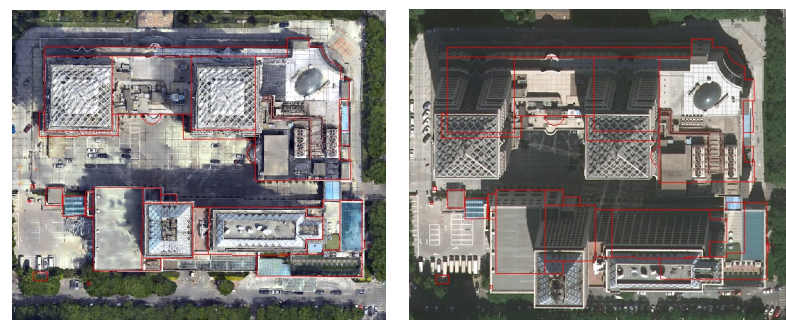

Figure 2. TDOM and DOM

2) Change detection combining DSM and TDOM. The DSM difference in Figure 1 was calculated simply by raster subtraction for the elevation change detection. The classic elevation changes, such as the building demolition and construction in urban areas, are easier to detect by setting a threshold value of $d D S M$. For some cases, there are obvious seasonal difference between original aerial data, the segmentation and tree extraction in dotted box could be applied to both TDOM images for reducing the crown influences of different seasons with the tree masks. The final result can be obtained by filtering the $d D S M$ with tree masks and the patch threshold including patch size and shape index, etc. (Yu, 2018).

\section{EXPERIMENTS AND DISCUSSION}

With the proposed change detection work flow, the experiments with two main traditional aerial sensors were carried out to verify the method.

\subsection{Change Detection with ADS 80 data}

Leica ADS80 is a push-broom Airborne digital aerial photogrammetry system. It integrates high-precision inertial navigation and orientation system and global positioning system. With 12000 pixels three-line array CCD scanning and professional single large aperture lens, in one flight it can obtain forward, nadir, backward images with $100 \%$ overlaps, to form continuous seamless panchromatic stereo images, RGB and infrared images (Wang et al., 2009). Since 2016, Beijing has launched an annual flight for DOM and DLG production with ADS80.

The data sets used are the Original ADS80 aerial images respectively obtained in 2016 and 2017. Both images were acquired during Oct. and Nov., so only the DSM difference was used for change detection. The study area was located near the $5^{\text {th }}$ ring road south in Beijing, which is the construction area of Fengtai District. The aerial triangulation of the ADS80 data in the format of L0 were processed by X-pro and PixGrid separately, and the results were acquired in the format of Level L1. The distribution of strips are different for 2016 and 2017 as Figure 3 (a) and Figure 3 (b). 


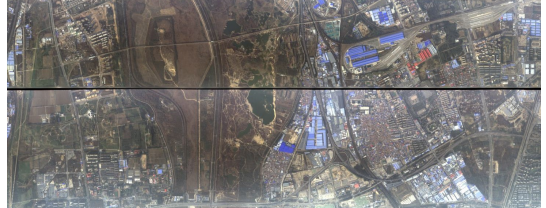

(a)

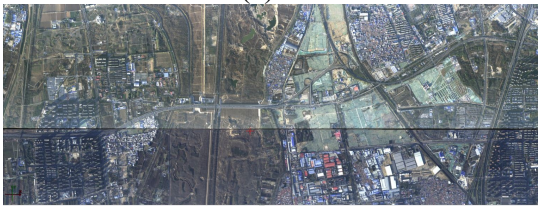

(b)

Figure 3. ADS 80 image strips for study area of 2016 and 2017 (a) ADS 80 image strips of 2016, (b) ADS 80 images strips of 2017

The most important advantage of SGM point cloud extraction is that the matched DSM data are irregular, non-smooth and no interpolation. Point clouds are more uniform and sparse in flat areas and increased appropriately in areas with mountainous area, which has a great effect on DTM generation by DSM filtering $(\mathrm{Xu}, 2019)$. The Dense DSM was generated based on nadir and backward viewing images of ADS80 data with SGM algorithm and the Census Transform (CT) was selected as matching cost value without considering setting the initial parallax value. Final DSM raster was interpolated with the resolution of $0.2 \mathrm{~m}$. DSM of 2017 is shown in Figure 4 (a) and the local result of red range of Figure 4 (a) is shown in Figure 4 (b).

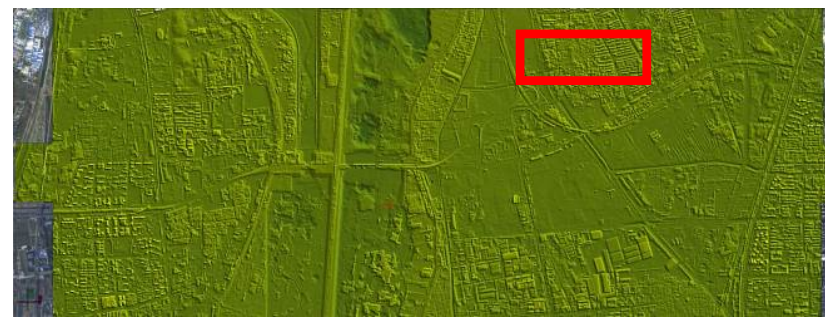

(a)

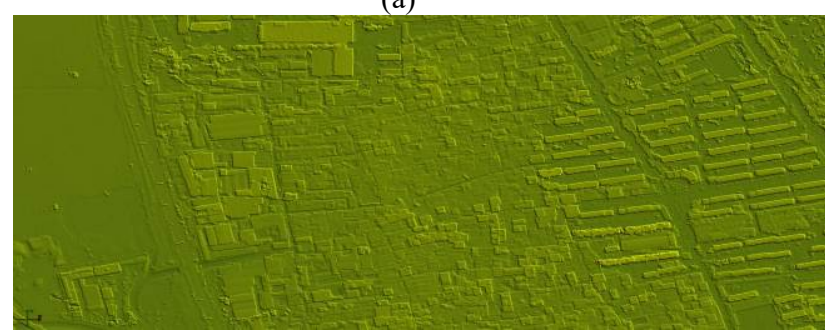

(b)

Figure 4. DSM result of 2017. (a) the whole range of DSM result of 2017, (b) local DSM of red rectangle range of (a)

The edges of building in built-up area easier to detect in Figure 4(b). Using the raster calculator, subtraction operation of the DSM data is performed to get the difference of DSM data. According to the requirements, the corresponding display intervals are designated and rendered according to different colors and the final results are as follows. Figure 4 shows the TDOM (Figure 5 (a) and Figure 5(b)) and final change detection result(Figure 5 (c) -Figure 5 (f)).

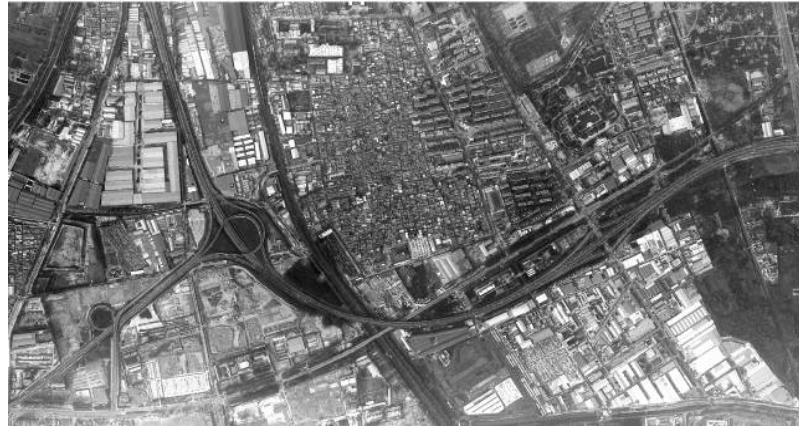

(a)

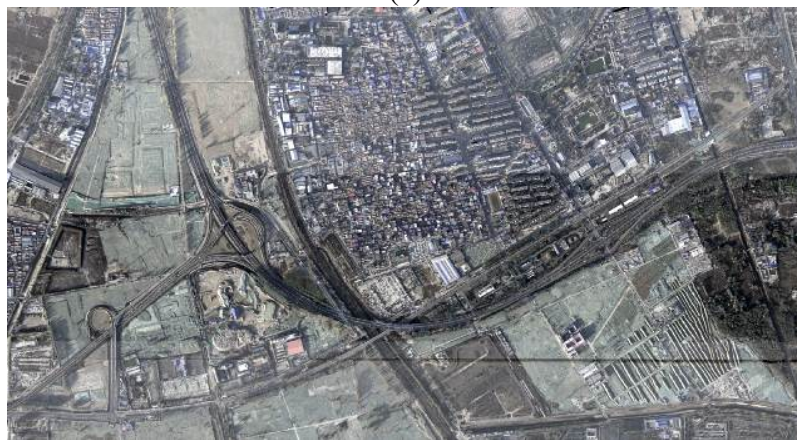

(b)

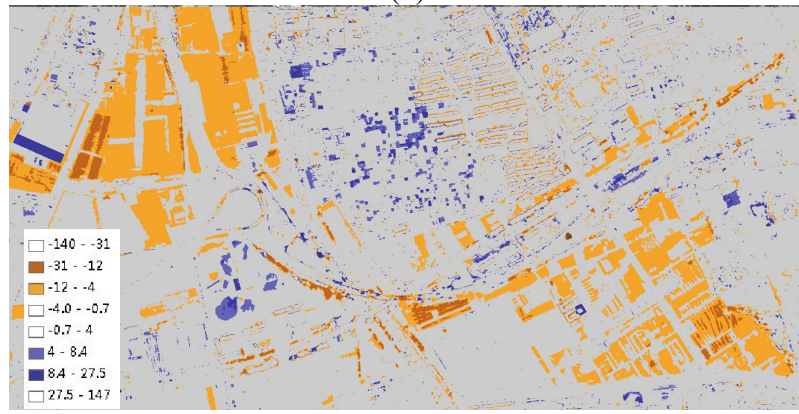

(c)
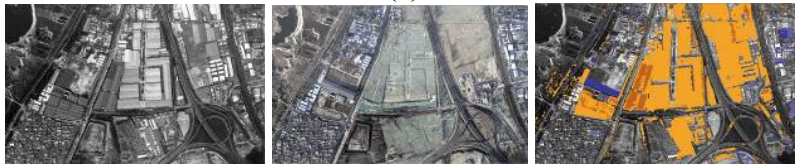

(d)
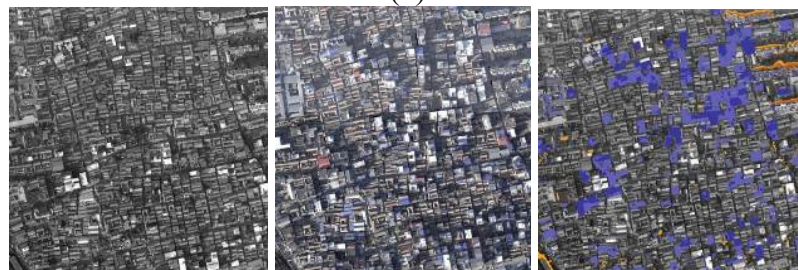

(e)
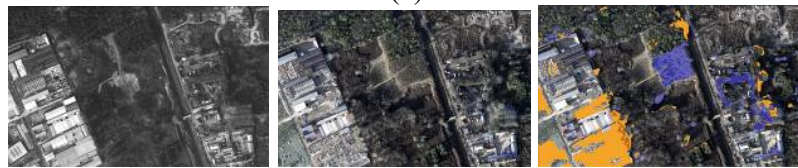

(f)

Figure 5. Change detection result with DSM. (a) TDOM of 2016, (b) TDOM of 2017, (c) DSM difference result between

2016 and 2017, (d) two period TDOM data and DSM

difference result of construction area, (e) two period TDOM data and DSM difference result of built-up area, (f) two period TDOM data and DSM difference result of regional greening 
By visual interpretation, the DSM differences could be observed and the edges of the change patches were relatively extracted with clear and regular, especially for buildings (Figure $5(\mathrm{e})$ ) and construction area (Figure $5(\mathrm{~d})$ ). It is easier to separate new construction (blue patches, including the storey increase) from the house demolition (yellow patches, including the storey decrease). The minimum area of the change patches could be $5 \mathrm{~m}^{2}$ when DSM is with the resolution of $0.2 \mathrm{~m}$, which was of great significance to the urban change detection. It was also worth noting that except the factory and residential area, for the regional greening, the significant elevation changes were also detected (Figure 5 (f)), which verified by field work that the method was also of great practical value for the monitoring of the non-planned deforestation and the planned afforestation implementation.

\subsection{Change Detection with frame digital aerial camera data}

An additional experiment was carried out with frame digital aerial camera data. The professional cameras, such as Digital Mapping Camera (DMC), UltraCam (UC), SWDC-4 and etc., are widely used because of the high imaging quality, wide coverage of single image and high accuracy to meet the requirements of large-scale mapping. In Beijing, before 2015, the frame camera were used in Beijing every year. The study area, the Dongcheng and Xicheng Districts of Beijing are he capital functional core areas and location of the historic city, covering 92 square kilometers. They are the most concentrated areas of Beijing's cultural relics and historic sites. There are many world cultural heritages, such as the Forbidden City and the Temple of Heaven. "Siheyuan" residences and the "hutongs" form the unique historic residential style, while, the high-rise buildings in Financial Street introduce the modern architectural elements to Beijing. At present, with policy of remediation, regulation and promotion, the protection and supervision of the Districts are particularly important. For the building demolition and construction supervision of Dongcheng and Xicheng District of Beijing between Oct. 2015 and May 2017. Both the UltraCam frame camera photos in 2015 and the RCD30 tilted aerial photos in 2017 were applied to extract the dense DSM and TDOM with the resolution of 0.2 $\mathrm{m}$, the result of 2015 and 2017 were shown in Figure 6 (a) and Figure $6(\mathrm{~b})$

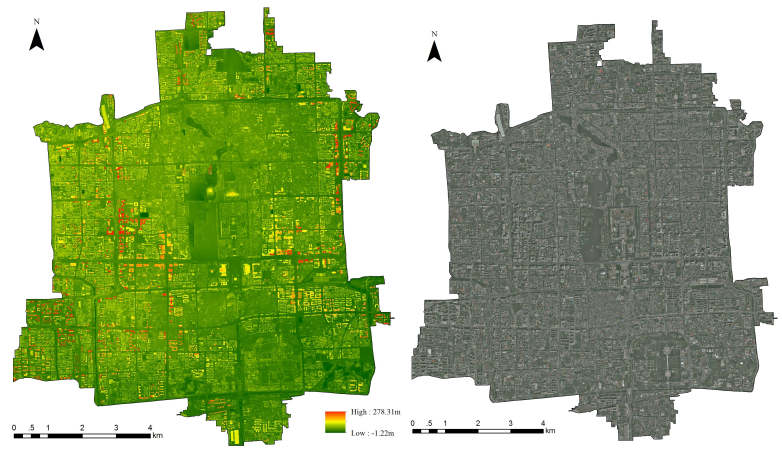

(a)
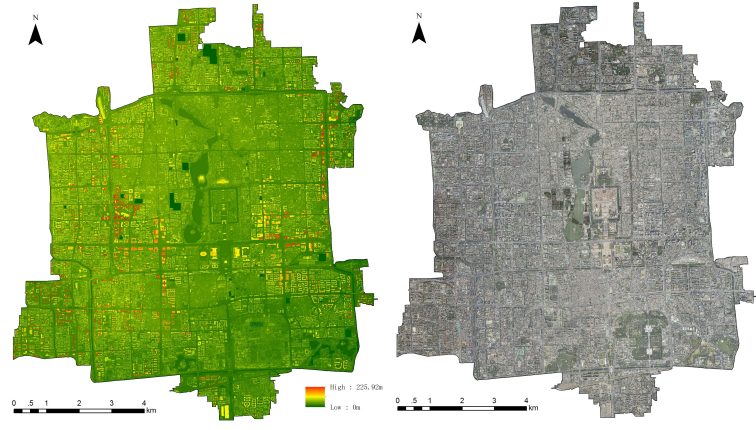

(b)

Figure 6. DSM and TDOM of 2015 and 2017. (a) DSM and TDOM of 2015, (b) DSM and TDOM of 2017

Because of the seasonal difference, tree masks were extracted as the pseudo-changes filter. 3-level segmentation and tree extraction of both TDOM based on Maximum Likeness Classifier (MLC) were carried out to reduce the change errors caused by the crown influence. By visual interpretation, the building height and area thresholds are set as $1.2 \mathrm{~m}$ and 5005 $\mathrm{m}^{2}$, respectively. The DSM difference result and the tree masks extracted from TDOM are shown in Figure 7 (a) and Figure 7 (b). The filtered result and visually extracted changes of the Dongcheng Distrct are shown in Figure 7 (c) and Figure 7 (d).

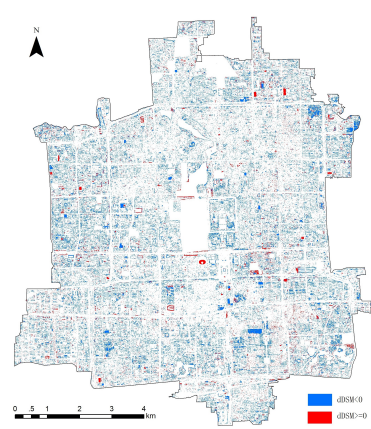

(a)

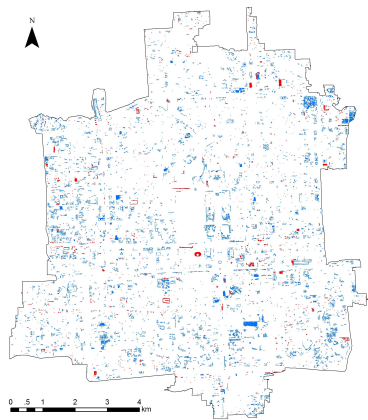

(c)

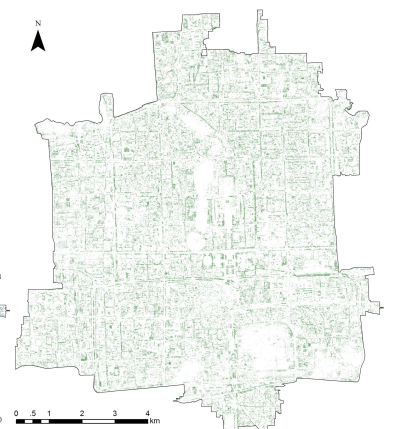

(b)

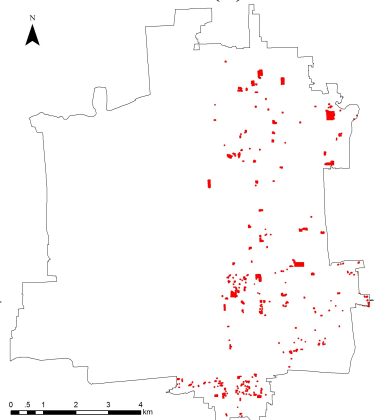

(d)
Figure 7. DSM difference and filter results. (a) DSM difference result between 2015 and 2017, (b) Tree masks from by segmentation and tree extraction with TDOM, (c) filtered result, (d) manually extracted changes of the Dongcheng District

The number of patches decreased from 395780 to 3915 before and after filtering with masks and threshold calculation. For Dongcheng Distract, the number of patches extracted automatically and identified artificially were 1702 and 549, respectively. Compared with the visual interpretation result, the method could effectively identify changes in plane and 
elevation, and all the visual identified changes are extracted automatically. From the result, $73 \%$ patches belong to building demolition. Some cases are given here to compare both the result.

The TDOM and DSM for the two periods are shown in Figure 8 (a)- (d), respectively. Especially for the constructing sites, the edges of the patch are regular, seen in Figure $8(\mathrm{~b})$. The number of patches extracted automatically is more than that identified manually, because the expression of patches extracted automatically is more detailed, and the range of the construction is usually selected manually (Figure 8(f)).

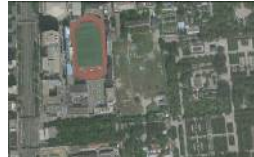

(a)

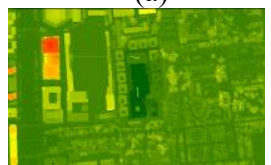

(d)

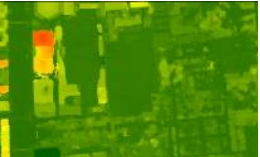

(b)

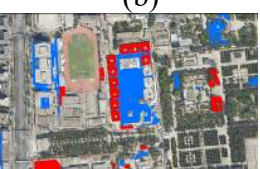

(e)

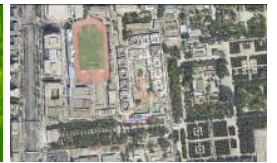

(c)

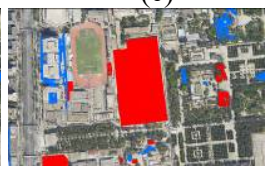

(f)
Figure 8. DSM difference and filter results. (a) TDOM of 2015, (b) DSM of 2015, (c) TDOM of 2017, (d) DSM of 2017, (e) automatically extracted change patches, (f) manually extracted change patches of the Dongcheng District

For the low-rise buildings (courtyard construction) concentrated in the 2nd Ring Road, there were always missing single building changes by visual interpretation, such as Figure 9 (a). In some cases, some buildings can not visually interpret whether there are storey increases or storey decreases, but change detection based on DSM and TDOM can effectively identify these changes and reduce missed detection (Figure 9 (b)). With the extracted changes of the above method, there were less missing patches with some pseudo-changes, which shown the method could assist checking the changes and improve the efficiency and accuracy of urban construction land change detection.
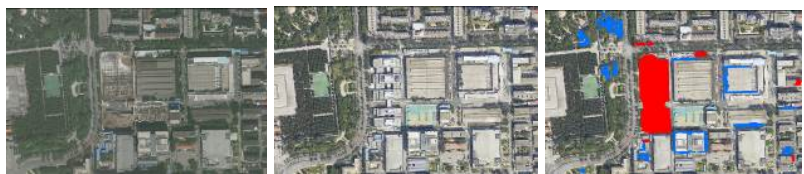

(a)
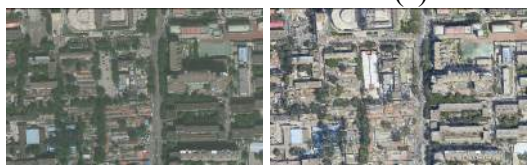

(b)

Figure 9. Low-rise building missing change patch cases. (a) TDOM of 2015 and 2017, single building patch extracted by change detection, but missed by visual interpretation, (b) TDOM of 2015 and 2017, storey increases change patch extracted by change detection, but unidentified by visual interpretation

The pseudo-changes exist in the final filtered result. After analysis, $15 \%$ of pseudo-changes are caused by matching difference between two periods or the quality between different DSM, and the size and shape index thresholds could be applied to filter these pseudo-changes. The other pseudo-changes are caused by SGM dense mismatching error. Compared with the DSM obtained by oblique photography, the DSM obtained by SGM intensive matching with traditional aerial photos has more mismatches. For example, Figure 10 (a) and Figure 10 (b) are the TDOM and DSM obtained by the 2015 frame aerial photographs with the heading overlap of $60 \%$ and side overlap of $30 \%$, and Figure 10 (c) and Figure 10 (d) are TDOM and DSM of oblique photography in 2017. The former is subject to shadows and low overlapping, after dense matching of SGM, some house collapse caused by mismatching. Through the difference of DSM data, errors are transmitted to the pseudochange (the red and blue patches in Figure 10 (e)). Through experiments, three-line push-broom ADS80 data with side overlap less than $40 \%$ and the satellite stereo data normally composed of two images will produce more similar errors during dense matching of SGM.

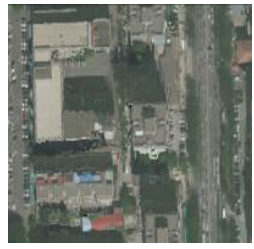

(a)

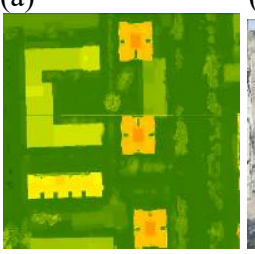

(d)

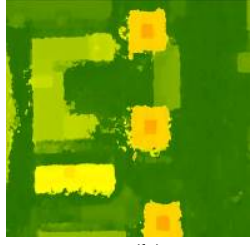

(b)

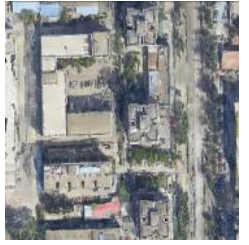

(c)

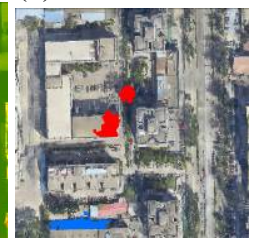

(e)

Figure 10. Pseudo-changes caused by SGM mismatching. (a) TDOM of 2015, (b) DSM of 2015, (c) TDOM of 2017, (d)

DSM of 2017, (e) pseudo-changes caused by SGM mismatching of frame digital aerial camera

\section{CONCLUSION AND PERSPECTIVES}

Through the method experiments with ADS80 and frame digital aerial camera data, it could be concluded that:

1) The surface changes of most urban construction land can be expressed as Changes in plane including position and shape, and changes in elevation. it is easier to identify the changes in plane with change detection between 2 period remote sensing images. However, for the changes in elevation caused by storey increase or decease, two-dimensional images are difficult to identify. Therefore, the difference of DSM is the best source applied to identify both the plane and elevation changes. Through the experiments, the changes of construction area, single building, regional greening could be identified and the minimum area of the change patches could be $5 \mathrm{~m}^{2}$ with the DSM resolution of $0.2 \mathrm{~m}$. The change patches of method could assist in visual inspection.

2) The pseudo-changes caused by vegetation crowns in different seasons could be extracted by TDOM segmentation and tree mask extraction. The pseudo-changes caused by matching and DSM accuracy could be removed by patch size and shape index threshold. Through the experiments, it is shown that most of the pseudo-changes are caused by the mismatching of SGM with traditional frame aerial photographs, 
which is mainly due to low overlap and shadow interference. It can be predicted that the improvement of SGM matching algorithm for non-tilt photography needs to be improved by increasing overlap degree from multi-period data and enhancing shadow texture of the images.

3) In this paper, ADS80 and UC frame data were applied, while the historical films, as the frame aerial photos, could also be explored for change detection based on DSM and TDOM. Like Beijing, many cities collect the aerial photos every year. The above methods can be extended to historically archived frame aerial films for long span, high-precision urban construction evaluation among 5-30 year. At present, the method has been applied to land cover change detection along the Cultural heritage of China Grand Canal (Beijing Section) with RC-10 aerial films acquired in 1970s - 1990s. In addition, with the improvement of the stereo acquisition capability and the acquisition cycle of the spaceborne sensors, the change detection experiment has been carried out with ZY-3 stereo images, more and more high-precision stereoscopic satellite images could be applied for the planning and supervision of the urban natural resources.

\section{ACKNOWLEDGEMENTS}

Acknowledgements of support of the Beijing Science and Technology Commission Project "Research and Development of Remote Sensing Monitoring System Platform for Cultural Heritage of China Grand Canal (Beijing Section)" (Project No. Z191100002419010) and support of the Science and Technology Commission Project of Tongzhou District "Tongzhou Urban Infrastructure Health Dynamic Monitoring System Platform Construction Project" (Project No. J2019CX034).

\section{REFERENCES}

Feng, Y., Pan, S., 2016: Land surface change monitoring based on DSM and remote sensing images, Science of Surveying and Mapping, 41(3), 44-48. doi.10.16251/j.cnki.10092307.2016.03.009.

Huang, X., Schneider, A., Friedl, M. A., Mapping sub-pixel urban expansion in China using MODIS and DMSP/OLS nighttime light, Remote Sensing of Environment, 175, 92-108.

Liu, Y., Zheng, X., Ai, G., 2018:UAV remote sensing true Digital Orthophoto Map high precision mapping, Bulletin of Surveying and Mapping, 2,83-88. doi.10.13474/j.cnki.112246.2018.0049.

Liu, Z., Zhang, J., Zhang, J., Fan, H., 2002: Change detection in urban areas based on DSM and image features. Remote Sensing technology and application, 17(10), 240-244.

Meng, X., Feng, W., 2018: Research on land cover change detection based on DSM technology. Geomatics \& Spatial Information Technology, 41(6), 101-103, 106.

Peng, D., Zhang, Y., Xiong, X., 2015: 3D Building change detection by combining LiDAR point clouds and aerial imagery. Geomatics and Information Science of Wuhan University, 40(4), 632-635, doi.10.13203/j.whugis20130325.
Shao, C., 2019: Research of high resolution remote sensing image DSM based on improved Semi-Global Matching algorithm. Beijing Surveying and Mapping, 33(6), 632-635, doi.19580/j.cnki.1007-3000.2019.06.005.

Wang, H., Wu, J., Feng, C., Wang, J., 2009: Leica ADS40/ADS80 digital aerial photogrammetry system, Bulletin of Surveying and Mapping, 10,73-74.

Wang, R., 2018. Spatio-temporal dynamics monitoring and driving factors analysis of built-up areas in Jiangxi, M.S., Wuhan University, Wuhan, China.

Wu, Y.,2019: Application of UAV oblique photography in automatic monitoring of illegal buildings. Geomatics \& Spatial Information Technology, 42(6), 205-209.

$\mathrm{Xu}$, S.,2019. Research on dense matching methods of aerial stereo image pairs, M.S., Wuhan University, Wuhan, China.

Yu, H., 2018: Research on DSM change detection and extraction method based on FME. Beijing Surveying and Mapping, 32(2), 218-220. doi.19580/j.cnki.10073000.2018.02.016. 